\title{
A step forward in disease modelling for mitochondrial diseases
}

\author{
Filippo Zambelli, Claudia Spits
}

Research Group Reproduction and Genetics, Vrije Universiteit Brussel, Laarbeeklaan 103, 1090, Jette, Belgium

Correspondence to: Filippo Zambelli. Research Group Reproduction and Genetics, Vrije Universiteit Brussel, Laarbeeklaan 103, 1090, Jette, Belgium. Email: zambelli.filippo@gmail.com; Claudia Spits. Research Group Reproduction and Genetics, Vrije Universiteit Brussel, Laarbeeklaan 103, 1090, Jette, Belgium. Email: claudia.spits@vub.be.

Provenance: This is an invited Editorial commissioned by Editor-in-Chief Zhizhuang Joe Zhao (Pathology Graduate Program, University of Oklahoma Health Sciences Center, Oklahoma City, USA).

Comment on: Lorenz C, Lesimple P, Bukowiecki R, et al. Human iPSC-derived neural progenitors are an effective drug discovery model for neurological mtDNA Disorders. Cell Stem Cell 2017;20:659-74.e9.

Received: 10 October 2017; Accepted: 27 October 2017; Published: 14 November 2017.

doi: $10.21037 /$ sci.2017.10.06

View this article at: http://dx.doi.org/10.21037/sci.2017.10.06

In vitro disease modelling has been always challenging, particularly so for multifactorial or late onset conditions, and for diseases affecting cell types that may be very hard to obtain and to keep in culture (such as for instance dopaminergic neurons in the case of Parkinson's disease). In multifactorial disease, the cellular models must be characterized by the complete set of mutations defining the disorder, and possibly kept in culture for a very long time to be able to observe the phenotype of late-onset conditions (1). In this sense, the discovery of human pluripotent stem cell (PSC), with their capacity to become any cell type of the adult body, has given a new impulse to the field of disease modelling (2). By now, numerous diseases have been in vitro modelled with embryonic stem cell (ESC) or induced pluripotent stem cell (iPSC) derivatives, with promising results (3). For instance, spinal muscular atrophy has been studied using iPSC-derived motor neurons, and the disease corrected by treatment with anti-apoptotic factors (4) and through gene editing (5). In Huntigton's disease, the inhibition of excessive mitochondrial fragmentation in iPSC-derived neurons could rescue defects in the mutant cells (6), and in amyotrophic lateral sclerosis, the pathogenic mechanisms were described thanks to the observations made on SOD1-mutated iPSC-based cellular models (7).

In the case of mitochondrial diseases, in vitro modelling suffers from an additional level of complication. Because of the multicopy nature of the mitochondrial genome, mutations can be found in a homoplasmic or heteroplasmic state (i.e., all mitochondrial genomes contain the mutation, or only a part of them). As such, it is difficult to evaluate the effect of a mutation in a heteroplasmic state, and to establish a threshold of heteroplasmy as from which the mutation affects the mitochondrial function (8). Furthermore, the same mutation at the same heteroplasmic level might cause a differential disease progression in different individuals, pointing towards a significant contribution of the nuclear DNA in modulating the effect of the mutation (9). One of the most commonly used tools to investigate the effects of mitochondrial mutations are cybrids, in which enucleated patient cells [(carrying their mitochondrial DNA (mtDNA)] are fused to immortalized cell-lines depleted of mtDNA (10). In these cases, there is a loss of nuclear DNA and mtDNA interactions, possibly modifying the behaviour of the diseased cells.

Recently, the group of Alessandro Prigione published an excellent paper, of great interest in this field of science, presenting a novel approach to mitochondrial disease modelling and drug discovery using multipotent neural progenitor cell (NPC) and neurons, derived from PSC (11).

In this work, the authors started by thoroughly validating their proposed model. The first step was to prove that these NPC are a representative proxy for human neural cells, especially regarding important mitochondrial features such as metabolism, membrane potential, calcium homeostasis and mtDNA inheritance. They first differentiated human iPSC and ESC towards NPC through exposure to small molecules. The authors performed their experiments with 
a variety of different lines to strengthen their findings and avoid accidental correlations. For instance, the iPSCs were derived with different reprogramming methods (episomal vectors and retroviral transduction) and different PSC lines (two ESCs and two iPSCs lines) were used to assess the model. The PSC-derived NPCs were shown to respond to calcium mobilization and acquire membrane polarization in a similar fashion to more mature differentiated neuronal-like cells. Moreover, the NPC relied on oxidative phosphorilation (OXPHOS) for energy production and were characterized by elongated mitochondria with welldefined cristae. Finally, these cells were retaining the mtDNA of their source, which did not undergo changes during reprogramming or differentiation. This last point is of particular importance, given the significant mtDNA genetic diversity seen in different iPSC generated from the same cellular source. The authors acknowledge that a thorough analysis of the genotype of the cells is imperative, especially since it is known from other studies that iPSC carrying certain mutations show an impaired differentiation and dysfunctional mitochondria, which could severely hamper the reliability of the study $(12,13)$. Also in this line, the authors suggest that, although they only worked on homoplasmic mutations, it should be possible to model in the same way the effect of heteroplasmic variants. Although this will probably be possible, again a lot of attention must be paid to the genotype of the reprogrammed cells. In fact, iPSCs have been found to change their heteroplasmic variants and their loads when compared to the source population due to the mosaic nature of mtDNA in cell culture (14).

In the second part of the study, the authors proceeded to use these cells as models for mitochondrial disease. They reprogrammed cells from three different patients with the same disease-causing mutation in the MT-ATP6 gene, in a homoplasmic state. Again, the authors used multiple iPSC lines with the same mtDNA mutation but from different patients, therefore with a different genetic background, to correlate the findings to the mutation itself, and avoiding confounding factors originating from one single individual. The NPC generated from these iPSC lines showed a defective ATP production, an altered mitochondrial membrane polarization and disrupted calcium homeostasis. Interestingly, the patient's fibroblasts and the cybrids also showed most of these features, but often to a lesser extent than the NPC. These findings illustrate the crucial importance of using the correct cell type to model the impact of a given mutation on a specific in vivo tissue. In the last part of their work, the authors carried out a screening of small molecules to find compounds able to revert the diseased phenotype of the mutated NPC. This screening yielded the PDE5 inhibitor avanafil as effective in partially rescuing the diseased phenotype induced by the MT-ATP6 mutation. Notably, the authors conducted the screening on 130 compounds, already FDA-approved for the treatment of other diseases. This strategy has the drawback that there is a limited number of compounds to test, but offers the great advantage of screening drugs that have already been declared safe. In this situation, if a compound is found to be effective, there is no need for the preclinical safety studies, which are risky, as drugs may be proven to be unsafe, expensive and time consuming (15). In this sense, the PDE5 inhibitor identified in the paper could hypothetically enter clinical studies in a very short time.

In summary, Lorenz et al. present a novel, wellcharacterized model for the study of mitochondrial disease. This approach was then subjected to a proof-of-concept by testing the impact of a specific mtDNA mutation on the cells, and to screen for drugs that correct the phenotype. Their work adds yet another successful approach to PSCbased disease modelling, a field of science that currently has a significant momentum and holds great promise for the understanding of the molecular mechanisms of disease and for effective drug discovery.

\section{Acknowledgements}

Filippo Zambelli is supported by the Methusalem grant to Karen Sermon, from the Vrije Universiteit Brussel.

\section{Footnote}

Conflicts of Interest: The authors have no conflicts of interest to declare.

\section{References}

1. Avior Y, Sagi I, Benvenisty N. Pluripotent stem cells in disease modelling and drug discovery. Nat Rev Mol Cell Biol 2016;17:170-82.

2. Park I, Arora N, Huo H, et al. Disease-specific induced pluripotent stem cells. Cell 2008;134:877-86.

3. Kim C. iPSC technology-Powerful hand for disease modeling and therapeutic screen. BMB Rep 2015;48:256-65.

4. Sareen D, Ebert AD, Heins BM, et al. Inhibition of apoptosis blocks human motor neuron cell death in a 
stem cell model of spinal muscular atrophy. PLoS One 2012;7:e39113.

5. Corti S, Nizzardo M, Simone C, et al. Genetic correction of human induced pluripotent stem cells from patients with spinal muscular atrophy. Sci Transl Med 2012;4:165ra162.

6. Guo X, Disatnik M, Monbureau M, et al. Inhibition of mitochondrial fragmentation diminishes Huntington' s disease - associated neurodegeneration. J Clin Invest 2013;123:5371-88.

7. Kiskinis E, Sandoe J, Williams LA, et al. Pathways disrupted in human ALS motor neurons identified through genetic correction of mutant SOD1. Cell Stem Cell 2014;14:781-95.

8. Inak G, Lorenz C, Lisowski $P$, et al. Concise review: induced pluripotent stem cell-based drug discovery for mitochondrial disease. Stem Cells 2017;35:1655-62.

9. Bénit P, El-Khoury R, Schiff M, et al. Genetic background influences mitochondrial function: modeling mitochondrial disease for therapeutic development. Trends Mol Med 2010;16:210-17.

doi: $10.21037 /$ sci.2017.10.06

Cite this article as: Zambelli F, Spits C. A step forward in disease modelling for mitochondrial diseases. Stem Cell Investig 2017;4:89.
10. Wilkins HM, Carl SM, Swerdlow RH. Cytoplasmic hybrid (cybrid) cell lines as a practical model for mitochondriopathies. Redox Biol 2014;2:619-31.

11. Lorenz C, Lesimple P, Bukowiecki R, et al. Human iPSCderived neural progenitors are an effective drug discovery model for neurological mtDNA Disorders. Cell Stem Cell 2017;20:659-74.e9.

12. Cherry AB, Gagne KE, McLoughlin EM, et al. Induced pluripotent stem cells with a mitochondrial DNA deletion. Stem Cells 2013;31:1287-97.

13. Hatakeyama TH, Katayama A, Komaki H, et al. Molecular pathomechanisms and cell-type-specific disease phenotypes of MELAS caused by mutant mitochondrial tRNA. Acta Neuropathol Commun 2015;3:52.

14. Kang E, Wang X, Tippner-Hedges R, et al. Age-related accumulation of somatic mitochondrial DNA mutations in adult-derived human iPSCs. Cell Stem Cell 2016;18:625-36.

15. McNeish J, Gardner JP, Wainger BJ, et al. From dish to bedside: lessons learned while translating findings from a stem cell model of disease to a clinical trial. Cell Stem Cell 2015;17:8-10. 